\title{
A PROBABILISTIC APPROACH TO THE TRACE AT THE BOUNDARY FOR SOLUTIONS OF A SEMILINEAR PARABOLIC PARTIAL DIFFERENTIAL EQUATION
}

\author{
JEAN-FRANÇOIS LE GALL \\ Université Paris VI, Laboratoire de Probabilités \\ 4, Place Jussieu, 75252 Paris Cedex 05, France
}

(Received August, 1996; Revised October, 1996)

\begin{abstract}
We use the path-valued process called the "Brownian snake" to investigate the trace at the boundary of nonnegative solutions of a semilinear parabolic partial differential equation. In particular, we characterize possible traces and in dimension one we prove that nonnegative solutions are in one-to-one correspondence with their traces at the origin. We also provide probabilistic representations for various classes of solutions.

This article is dedicated to the memory of Roland L. Dobrushin.

Key words: Parabolic Partial Differential Equation, Boundary Trace, Brownian Snake, Probabilistic Representation, Additive Functionals, Polar Sets, Maximal Solutions.
\end{abstract}

AMS (MOS) subject classifications: $35 \mathrm{~K} 55,60 \mathrm{~J} 45$.

\section{Introduction and Statement of the Results}

The goal of this work is to develop a probabilistic approach for studying the trace at the boundary of positive solutions to the semilinear parabolic equation

$$
\frac{\partial u}{\partial t}=\frac{1}{2} \Delta u-u^{2}
$$

This approach has been inspired by our previous work [14] and the recent paper of Dynkin and Kuznetsov [7], which both dealt with the trace at the boundary for related semilinear elliptic partial differential equations. Our main probabilistic tool is the path-valued process called the Brownian snake, whose connections with semilinear partial differential equations have been investigated in several recent papers $[10,13,14]$. Since the Brownian snake is closely related to the super-Brownian motion, part of these connections can be viewed as a reformulation of Dynkin's important work on the relation between superprocesses and partial differential equations [3-5]. However, we think that the Brownian snake is more tractable, although less general, for certain applications. In particular, it is not clear how to derive the results of [14] or of Section 4 of the present work, using only the theory of superprocesses. On the other hand, it is very plausible that superprocesses can be applied to extend a significant part of the present work to more general equations where the nonlinear term $u^{2}$ is replaced by $u^{\alpha}$ for $1<\alpha \leq 2$.

The problem of the trace at the boundary for semilinear elliptic or parabolic equations of type (1) has also been studied recently by analytic methods. See in particular, Marcus and Véron [15]. 
Very recently, as the final version of this work was in preparation, we received the note [16], which announces results that generalize some of the statements below (Theorem 1 and the analytic part of Theorem 4) to equations of form (1) with a nonlinearity $u^{p}$ instead of $u^{2}$, for any $p>1$. Nonetheless, we feel that it is worth developing the probabilistic approach, which in the case $p=2$ gives slightly more precise results (in contrast to [16], we are able to characterize all possible traces; see Proposition 2 below) and also yields explicit probabilistic formulas for the solutions.

Let us now state our main results. For $y \in \mathbb{R}^{d}$ and $r>0$, we denote by $B(y, r)$ the open ball of radius $r$ centered at $y$.

Theorem 1: Let $\Omega$ be a domain in $\mathbb{R}^{d}$ and let $u \in C^{1,2}((0, \infty) \times \Omega)$ be a nonnegative solution of $(1)$ in $(0, \infty) \times \Omega$. Set

$$
\Lambda=\left\{y \in \Omega ; \forall r>0, \lim _{t \rightarrow 0} \int_{B(y, r)} u(t, z) d z=\infty\right\} .
$$

There exists a Radon measure $\nu$ on $\Omega \backslash \Lambda$ such that, for every $\varphi \in C_{c}(\Omega \backslash \Lambda)$,

$$
\langle\nu, \varphi\rangle=\lim _{t \rightarrow 0} \int_{\Omega \backslash \Lambda} u(t, z) \varphi(z) d z .
$$

The pair $(\Lambda, \nu)$ is called the trace of $u$.

The method of proof gives precise information about the behavior of $u$ near $(0, y)$, when $y \in$ $\Omega \backslash \Lambda$. See Lemma 6 and the remark following the proof of Theorem 1 in Section 3 .

Our second result gives a characterization of possible traces of a solution. It is obviously analogous to Theorem 1.3 of Dynkin and Kuznetsov [7]. In this statement, "polar" means "polar with respect to Brownian motion in $\mathbb{R}^{d}$."

Proposition 2: Let $\Lambda$ be a closed subset of $\Omega$ and let $\nu$ be a Radon measure on $\Omega \backslash \Lambda$. In order that the pair $(\Lambda, \nu)$ be the trace of a nonnegative solution of $(1)$ in $(0, \infty) \times \Omega$, it is necessary and sufficient that the following conditions hold:

(a) $\quad \nu$ does not charge polar sets.

(b) Let $\operatorname{Ex}(\nu)=\{y \in \Lambda, \forall r>0, \nu(B(y, r))=\infty\}$ be the set of explosion points of $\nu$ and let $H$ be a polar subset of $\Lambda$ such that $\Lambda \backslash H$ is closed and $H \cap E x(\nu)=\emptyset$. Then $H=\emptyset$.

Proposition 2 resolves the problem of the existence of a solution with a given trace. We now address the uniqueness problem when $\Omega=\mathbb{R}^{d}$. We use the probabilistic representation of solutions involving the Brownian snake $\left(W_{s}, s \geq 0\right)$ under its excursion measures $\mathbb{N}_{x}, x \in \mathbb{R}^{d}$. A brief presentation of these probabilistic objects is given in Section 2 below. Here, we simply note that, under $\mathbb{N}_{x}$, for every $s \geq 0, W_{s}:\left[0, \zeta_{s}\right] \rightarrow \mathbb{R}^{d}$ is a finite path in $\mathbb{R}^{d}$ started at $x$ and with "lifetime" $\zeta_{s}$. The lifetime process $\left(\zeta_{s}, s \geq 0\right)$ is distributed under $\mathbb{N}_{x}$ according to the Itô measure of positive excursions of a linear Brownian motion. For every $t \geq 0$, we set

$$
\varphi_{t}=\left\{W_{s}(t), s \geq 0, \zeta_{s} \geq t\right\},
$$

which corresponds to the values at time $t$ of all paths $W_{s}$ with lifetime $\zeta_{s} \geq t$. Let $\nu$ be a finite measure on $\mathbb{R}^{d}$ not charging polar sets. For every $t>0$, we can consider the finite measure on paths with lifetime $t$ defined by

$$
\mu_{x, t}(d w)=\int \nu(d y) p_{t}(x, y) P_{x y}^{t}(d w)
$$

where $p_{t}(x, y)$ is the Brownian transition density, and $P_{x y}^{t}$ denotes the law of the Brownian bridge from $x$ to $y$ in time $t$. Following [2], we can associate with the measure $\mu_{t, x}$ an additive functional of the Brownian snake with initial point $x$. We denote by $A^{t, \nu}=\left(A_{s}^{t, \nu}, s \geq 0\right)$ this additive functional. By a monotonicity argument, the definition of $A^{t, \nu}$ can be extended to the case when $\nu$ is a countable sum of finite measures not charging polar sets. 
Proposition 3: Suppose that $\Omega=\mathbb{R}^{d}$ and let $(\Lambda, \nu)$ be a pair satisfying the conditions of Proposition 2. Then there exists a maximal nonnegative solution of $(1)$ with trace $(\Lambda, \nu)$, and it is given by the probabilistic formula

$$
u(t, x)=2 \mathbb{N}_{x}\left(\varphi_{t} \cap \Lambda \neq \emptyset\right)+2 \mathbb{N}_{x}\left(1_{\left\{\varphi_{t} \cap \Lambda=\emptyset\right\}}\left(1-\exp \left(-A_{\infty}^{t, \nu / 2}\right)\right)\right) .
$$

If $\Lambda$ is polar, $u$ is the unique nonnegative solution with trace $(\Lambda, \nu)$. On the other hand, if $d \geq 2$, and if $(\Lambda, 0)$ is a possible trace, with $\Lambda \neq \emptyset$, then there exist infinitely many solutions with the trace $(\Lambda, 0)$.

For a general choice of $(\Lambda, \nu)$ in dimension of $d \geq 2$, it is not easy to decide whether there is a unique associated solution. A natural guess would be that uniqueness holds if and only if $\Lambda=\operatorname{Ex}(\nu)$, but we were unable to prove this.

In dimension $d=1$, uniqueness always holds. This follows from the next theorem, where the probabilistic representation of solutions is made somewhat more explicit by the following considerations. For every $t>0$, denote by $L^{t}=\left(L_{s}^{t}, s \geq 0\right)$ the local time process of $\left(\zeta_{s}, s \geq 0\right)$ at level $t$. Then let $X_{t}$ be the random measure on $\mathbb{R}^{d}$ supported on $S_{t}$ defined by

$$
\left\langle X_{t}, \varphi\right\rangle=\int_{0}^{\infty} d L_{s}^{t} \varphi\left(W_{s}(t)\right) .
$$

Then [9] the distribution of $\left(X_{t}, t>0\right)$ under $\mathbb{N}_{x}$ is the canonical measure of super-Brownian motion with initial point $x$. When $d=1$, it follows from well-known results (see e.g., Sugitani [18], Theorem 1) that the measures $X_{t}$ are absolutely continuous, and, more precisely, we may write $X_{t}(d y)=Y_{t}(y) d y$ where the process $\left(Y_{t}(y) ; t>0, y \in \mathbb{R}\right)$ is jointly continuous. With this notation at hand, we can state our last result, which is analogous to the main result of [14].

Theorem 4: Let $d=1$ and $\Omega=\mathbb{R}$. The formula

$$
u(t, x)=2 \mathbb{N}_{x}\left(\varphi_{t} \cap \Lambda \neq \emptyset\right)+2 \mathbb{N}_{x}\left(1_{\left\{\varphi_{t} \cap \Lambda=\emptyset\right\}}\left(1-\exp -\frac{1}{2} \int \nu(d y) Y_{t}(y)\right)\right)
$$

gives a one-to-one correspondence between nonnegative solutions of $(1)$ and pairs $(\Lambda, \nu)$, where $\Lambda$ is a closed subset of $\mathbb{R}$ and $\nu$ is a Radon measure on $\mathbb{R} \backslash \Lambda$. If $u$ is given by formula (3), the trace of $u$ is $(\Lambda, \nu)$.

The paper is organized as follows. In Section 2, we recall the basic facts about the Brownian snake, its additive functionals and the connections with solutions of (1). Several results of this section are valid in a much greater generality, but we limited ourselves to those facts that are needed in the proofs of the following sections. In Section 3, we give the proof of Theorem 1 and Propositions 2 and 3. Several arguments of this section are inspired from [7], although our definition of the trace is different and more analytic in the spirit of [15] and [16]. Finally, in Section 4, we prove Theorem 4 following ideas from [14].

\section{Preliminaries}

\subsection{Analytic Preliminaries}

In view of the probabilistic representation, it will be preferable to deal with a slightly modified form of equation (1). We shall be interested in solutions of the equation

$$
\frac{\partial u}{\partial t}+\frac{1}{2} \Delta u=2 u^{2}
$$

in $(-\infty, 0) \times \Omega$. Up to a trivial scale parameter, the change of variables $t \rightarrow-t$ reduces the study of (1) to that of (4). Rather than proving the results stated in the introduction, we shall prove below the equivalent statements concerning (4). As we shall deal only with nonnegative solu- 
tions, the word "solution" will always mean "nonnegative solution". A solution of (4) in the domain $D$ of $\mathbb{R} \times \mathbb{R}^{d}$ is thus a nonnegative function $u \in C^{1,2}(D)$ such that (4) holds pointwise in $D$.

Brownian motion in $\mathbb{R}^{d}$ will be denoted by $\xi$. For every $(t, x) \in \mathbb{R} \times \mathbb{R}^{d}$, we will denote by $P_{t, x}$ the probability measure under which $\xi$ starts at $x$ at time $t\left(\xi_{r}\right.$ is thus defined for $\left.r \geq t\right)$.

Let $D$ be a bounded domain in $\mathbb{R} \times \mathbb{R}^{d}$. We denote by $\tau$ the exit time of $\xi$ from $D: \tau=$ $\inf \left\{r ;\left(r, \xi_{r}\right) \notin D\right\}$. A measurable subset $H$ of $\partial D$ is called total if $P_{t, x}\left(\left(\tau, \xi_{\tau}\right) \in H\right)=1$, for every $(t, x) \in D$. The following maximum principle for solutions of (4) can be found, in a more general form, in the appendix of Dynkin [4].

Maximum principle: Let $u_{1}$ and $u_{2}$ be two (nonnegative) solutions of (4) in $D$. Assume that $u_{1}-u_{2}$ is bounded above and that there exists a total subset $H$ of $\partial D$ such that for every $(r, y) \in H$,

Then $u_{1} \leq u_{2}$.

$$
\limsup _{D \ni(t, x) \rightarrow(r, y)}\left(u_{1}(t, x)-u_{2}(t, x)\right) \leq 0 .
$$

\subsection{The Brownian snake}

It will be convenient to work in a time-inhomogeneous setting as described in [13], Section 2.1. Therefore, we slightly extend the notation of the introduction as follows. For every fixed $(t, y) \in \mathbb{R} \times \mathbb{R}^{d}$, we denote by $\mathcal{W}_{t, x}$ the space of all finite continuous paths $w:[t, \zeta] \rightarrow \mathbb{R}^{d}$ such that $w(t)=x$ (here $\zeta=\zeta_{w}$ can be any real number in $[t, \infty)$ ). We denote by $\left(W_{s}, s \geq 0\right)$ the Brownian snake in $\mathcal{W}_{t, x}$ and by $\mathbb{N}_{t, x}$ its excursion measure away from the trivial path in $\mathcal{W}_{t, x}$ with $\zeta=t$ (see [13], Section 2.1). Under $\mathbb{N}_{t, x}$, each $W_{s}:\left[t, \zeta_{s}\right] \rightarrow \mathbb{R}^{d}$ is a finite path in $\mathbb{R}^{d}$, started from $x$ at time $t$ and stopped at time $\zeta_{s}$. The distribution of $\left(\zeta_{s}, s \geq 0\right)$ under $\mathbb{N}_{t, x}$ is the Itô measure of excursions of linear Brownian motion above level $t$. Informally, the path $W_{s}$ extends itself by adding little pieces of Brownian motion when $\zeta_{s}$ increases, and erases itself when $\zeta_{s}$ decreases.

For $w \in \mathbb{W}_{t, x}$, we also denote by $\mathbb{P}_{w}^{*}$ the law of the Brownian snake started at $w$ and stopped when it first hits the trivial path of $\mathcal{W}_{t, x}$ (equivalently when $\zeta_{s}$ first hits $t$ ).

The range and graph of the Brownian snake are defined respectively as

$$
\mathscr{B}_{0}=\left\{W_{s}(r) ; s \geq 0, t \leq r \leq \zeta_{s}\right\}, \quad \mathcal{G}=\left\{\left(r, W_{s}(r)\right) ; s \geq 0, t \leq r \leq \zeta_{s}\right\} .
$$

Note that $\mathscr{R}$ and $\mathcal{G}$ are compact connected subsets of $\mathbb{R}^{d}$ and $\mathbb{R} \times \mathbb{R}^{d}$, respectively. It is easy to prove that for every $\varepsilon>0$,

$$
\mathbb{N}_{t, x}\left(\Re \cap B(x, \varepsilon)^{c} \neq \emptyset\right)=c_{d} \varepsilon^{-2}, \mathbb{N}_{t, x}\left(g \cap[t+\varepsilon, \infty) \times \mathbb{R}^{d} \neq \emptyset\right)=(2 \varepsilon)^{-1},
$$

where $c_{d}$ is a positive constant (see e.g., [10]).

For $t^{\prime}>t$, we also set

$$
\mathscr{R}_{\left[t, t^{\prime}\right]}=\left\{W_{s}(r) ; s \geq 0, t \leq r \leq t^{\prime} \wedge \zeta_{s}\right\} .
$$

Let $r>0$ and $\psi_{r}(\varepsilon)=\mathbb{N}_{t, x}\left(\mathscr{R}_{[t, t+\varepsilon]} \cap B(x, r)^{c} \neq \emptyset\right)$. We shall frequently use the fact that

$$
\lim _{\varepsilon \downarrow 0} \psi_{r}(\varepsilon)=0
$$

One way to derive (5) is to check from the Kolmogorov lemma that the paths $W_{s}, s>0$, satisfy a uniform Hölder condition in the variable $t$. In fact, results much more precise than (5) are known. See in particular, Theorem 3.3 of [1].

As in Section 1, we write $\varphi_{r}=\left\{W_{s}(r): s \geq 0, \zeta_{s} \geq r\right\}$, for every $r \geq t$. Note that $[r\} \times \varphi_{r}=g \cap\left(\{r\} \times \mathbb{R}^{d}\right)$. By a result of Dynkin [4], if $H$ is a Borel subset of $\mathbb{R}^{d}$ and $r>t$, $\mathbb{N}_{t, x}\left(\varphi_{r} \cap H \neq \emptyset\right)$ is positive if and only if $H$ is not polar. This fact explains the relevance of polar sets in our main results. 


\subsection{Exit measures}

We now consider a domain $D$ in $\mathbb{R} \times \mathbb{R}^{d}$ of the type $(-\infty, a) \times \Omega$. As previously, we denote by $\tau$ the first exit time of Brownian motion from $D$ and we also write $\tau(w)$ for the first exit time from $D$ of a finite path $w$.

Assuming that $(t, x) \in D$, we can define the exit measure of the Brownian snake from $D$ under the excursion measure $\mathbb{N}_{t, x}$ (see [13], Section 2.2 or [10]). This measure, denoted by $X^{D}$, is a finite measure supported on $\left\{\left(\tau\left(W_{s}\right), W_{s}\left(\tau\left(W_{s}\right)\right)\right) ; s \geq 0, \tau\left(W_{s}\right)<\infty\right\} \subset \partial D$. In the special case $D=D_{a}=(-\infty, a) \times \mathbb{R}^{d}$, we can write $X^{D_{a}}=\delta_{a} \otimes X_{a}$, where $X_{a}$ is a finite measure on $\mathbb{R}^{d}$. This definition is consistent with the notation of Section 1.

Let $g$ be a nonnegative measurable function on $\partial D$, which is bounded on the sets $\partial D \cap\left([r, \infty) \times \mathbb{R}^{d}\right)$. By applying Theorem 4.2 of [10] to the special case when the underlying spatial motion is a space-time Brownian motion, we get that the function

$$
u(t, x)=\mathbb{N}_{t, x}\left(1-\exp -\left\langle X^{D}, g\right\rangle\right),(t, x) \in D,
$$

solves the integral equation

$$
u(t, x)+2 E_{t, x} \int_{t}^{\tau} d r u\left(r, \xi_{r}\right)^{2}=E_{t, x}\left(g\left(\tau, \xi_{\tau}\right)\right) .
$$

From this integral equation and the classical connections between (space-time) Brownian motion and the heat equation, we easily deduce that the function $u$ solves (4) in $D$.

Conversely, solutions of (4) can be represented in the previous form.

Mean value property: Let $u$ be a solution of (4) in $D$. Let $D^{\prime}$ be a subdomain of $D$ of the form $D^{\prime}=\left(-\infty, a^{\prime}\right) \times \Omega^{\prime}$, where $a^{\prime}<a$ and $\Omega^{\prime}$ is a relatively compact subdomain of $\Omega$. Then, for every $(t, x) \in D^{\prime}$

$$
u(t, x)=\mathbb{N}_{t, x}\left(1-\exp -\left\langle X^{D^{\prime}}, u\right\rangle\right) .
$$

This follows from the maximum principle applied to $u$ and to the function $v(t, x)=\mathbb{N}_{t, x}\left(1-\exp -\left\langle X^{D^{\prime}}, u\right\rangle\right)$ in the domain $\left(r, a^{\prime}\right) \times \Omega^{\prime}, r<a^{\prime}$. We have seen that $v$ solves (4) in $D^{\prime}$ and from the integral equation satisfied by $v$, it is easy to verify that $v$ has boundary value $u$ on a total subset of $\partial D^{\prime}$.

In the special case $\Omega=\mathbb{R}^{d}$, we can take $\Omega^{\prime}=B(0, R)$ and then let $R \rightarrow \infty$ to obtain

for $t<r<a$.

$$
u(t, x)=\mathbb{N}_{t, x}\left(1-\exp -\left\langle X_{r}, u(r, \cdot)\right\rangle\right)
$$

Remark: The previous mean value property can be stated in a much more general form.

\subsection{Additive functionals}

We now take $D=(-\infty, 0) \times \Omega$ for simplicity. In order to construct more general solutions of (4), we introduce additive functionals of the Brownian snake. Let $\nu$ be a finite measure on $\Omega$. We first assume that $\nu$ has a finite energy in the classical potential-theoretic sense:

$$
\iint \nu(d y) \nu(d z) f_{d}(|y-z|)<\infty
$$

where $f_{1}(r)=1, f_{2}(r)=\log _{+}(1 / r)$, and $f_{d}(r)=r^{2-d}$ if $d \geq 3$. If $t<0$ and $x, y \in \Omega$, we denote by $P_{x y}^{t 0}$ the law of the Brownian bridge started from $x$ at time $t$ and conditioned to be at $y$ at time 0 . We can view $P_{x y}^{t 0}$ as a probability measure on the set $\left\{w \in \mathcal{W}_{t, x}, \zeta=0\right\}$. We also denote by $P_{x y}^{t 0, \Omega}$ the law of the bridge conditioned to remain inside $\Omega$. We set 


$$
\mu_{t, x}(d w)=\int \nu(d y) p_{-t}^{\Omega}(x, y) P_{x y}^{t 0, \Omega}(d w)
$$

where $p_{r}^{\Omega}(x, y)$ stands for the transition density of Brownian motion killed when it exits $\Omega$. We can interpret $\mu_{t, x}$ as the law of the $h$-transform of a space-time Brownian motion in $D$ started at $(t, x)$ corresponding to the harmonic function $h(t, x)=\int \nu(d y) p_{-t}^{\Omega}(x, y)$.

The energy of $\mu_{t, x}$ with respect to the Brownian snake in $\mathcal{W}_{t, x}$ is easily computed from [12] Proposition 1.1:

$$
\varepsilon\left(\mu_{t, x}\right)=2 E_{t, x} \int_{t}^{\tau} d r h\left(r, \xi_{r}\right)^{2}=2 \int_{t}^{0} d r \int_{\Omega} d y p_{r-t}^{\Omega}(x, y)\left(\int \nu(d z) p_{-r}^{\Omega}(y, z)\right)^{2} .
$$

Thus,

$$
\varepsilon\left(\mu_{t, x}\right) \leq 2 \iint \nu(d z) \nu\left(d z^{\prime}\right) F_{t, x}\left(z, z^{\prime}\right)
$$

where $F_{t, x}\left(z, z^{\prime}\right)=2 \int_{t}^{0} d r \int_{\mathbb{R}^{d}} d y p_{r-t}(x, y) p_{-r}(y, z) p_{-r}\left(y, z^{\prime}\right) . \quad$ Elementary estimates give the bound $F_{t, x}\left(z, z^{\prime}\right) \leq C(t) f_{d}\left(\left|z-z^{\prime}\right|\right)$, with a constant $C(t)<\infty$ depending only on $t$. Therefore our assumption on $\nu$ guarantees that $\varepsilon\left(\mu_{t, x}\right)<\infty$.

We can then use [2] Theorem 5 to construct the continuous additive functional associated with the measure $\mu_{t, x}$, which we denote by $A^{\nu, \Omega}=\left(A_{s}^{\nu, \Omega}, s \geq 0\right.$ ) (we drop $(t, x)$ in the notation since it is understood that we work under the measure $\left.\mathbb{N}_{t, x}\right)$. For any nonnegative measurable function $F$ on $W_{t, x}$, we have

$$
\mathbb{N}_{t, x}\left(\int_{0}^{\infty} d A_{s}^{\nu, \Omega} F\left(W_{s}\right)\right)=\int \mu_{t, x}(d w) F(w)
$$

An approximation for $A^{\nu, \Omega}$ can also be given as follows. For every $r>0$, set $D_{r}=(-\infty, r) \times \Omega$, and make the convention that $h=0$ on $(-\infty, 0) \times \partial \Omega$. Then,

$$
A_{\infty}^{\nu, \Omega}=\lim _{r \uparrow 0}\left\langle X^{D}, h\right\rangle
$$

in $L^{2}\left(\mathbb{N}_{t, x}\right)$ (compare with [13], Section 4.4). This follows from standard energy calculations: for $t<r<0,\left\langle X^{D} r, h\right\rangle=A_{\infty}^{\nu, \Omega, r}$, where $A^{\nu, \Omega, r}$ is the additive functional whose associated measure $\mu_{t, x, r}$ is the image of $\mu_{t, x}$ under the mapping $w \rightarrow w_{\mid[t, r]}$. Then observe that $\mu_{t, x, r}$ converges to $\mu_{t, x}$ in the energy norm.

It is easy to extend the previous construction to the case when $\nu$ is a finite measure that does not charge polar sets. By a classical result, we may write $\nu=\lim \uparrow \nu_{n}$, where the measures $\nu_{n}$ have a finite energy. Then, we may define $A_{s}^{\nu, \Omega}=\lim \uparrow A_{s}^{\nu}{ }^{,}, \Omega$, for every $s \geq 0, \mathbb{N}_{t, x}$ a.e. By (6), $\mathbb{N}_{t, x}\left(A_{\infty}^{\nu, \Omega}\right)=h(t, x)<\infty$, so that $A_{\infty}^{\nu, \Omega}<\infty, \mathbb{N}_{t, x}$ a.e. In addition, from the time-reversal invariance property of additive functions ([2] Theorem 5), we have also $A_{\infty}^{\nu, \Omega}-A_{s}^{\nu, \Omega}=$ $\lim \uparrow\left(A_{\infty}^{\nu}{ }_{n}, \Omega-A_{s}^{\nu} n^{,},\right)$, for every $s \geq 0, \mathbb{N}_{t, x}$ a.e. It follows that the convergence of $A_{s}^{\nu}{ }^{\nu}, \Omega$ to $A_{s}^{\nu, \Omega}$ is uniform in $s \geq 0$, and we easily conclude that $A^{\nu, \Omega}$ is also a continuous additive functional for which formula $(6)$ still holds.

Proposition 5: Let $\nu$ be a finite measure on $\Omega$ that does not charge polar sets. For every $(t, x) \in(-\infty, 0) \times \Omega$, set

$$
u_{\nu, \Omega}(t, x)=\mathbb{N}_{t, x}\left(1-\exp -A_{\infty}^{\nu, \Omega}\right) .
$$

The function $u_{\nu, \Omega}$ is the unique nonnegative solution of the integral equation

$$
u(t, x)+2 E_{t, x} \int_{t}^{\tau} d r u\left(r, \xi_{r}\right)^{2}=\int \nu(d y) p_{-t}^{\Omega}(x, y) .
$$


In particular, $u_{\nu, \Omega}$ solves (4) in $(-\infty, 0) \times \Omega$. In addition, for any bounded continuous function $\varphi$ on $\Omega$, we have

$$
\lim _{t \uparrow 0} \int_{\Omega} d x \varphi(x) u_{\nu, \Omega}(t, x)=\int \nu(d x) \varphi(x) .
$$

Proof: Write $u=u_{\nu, \Omega}$ and $h(t, x)=\int \nu(d y) p_{-t}^{\Omega}(x, y)$ as previously. We use the strong Markov property under $\mathbb{N}_{t, x}[10]$ and the fact that $A^{\nu, \Omega}$ is an additive functional to obtain

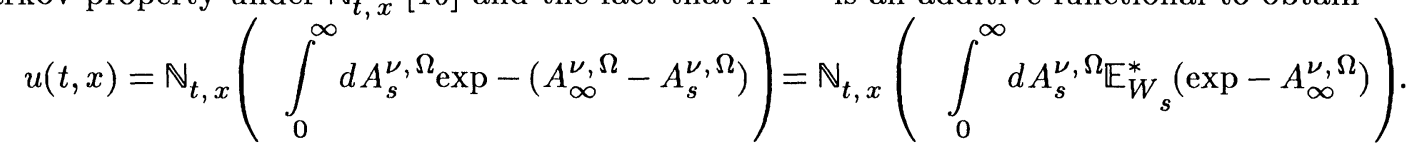

By (6) and the form of the measure $\mu_{t, x}$, we know that $d A_{s}^{\nu, \Omega}$ a.e. $W_{s}$ is a finite path in $\Omega$ started at time $t$ and stopped at time 0. We can use [10] Proposition 2.5 to compute $\mathbb{E}_{W_{s}}^{*}\left(\exp -A_{\infty}^{\nu, \Omega}\right)$. Approximation (7) allows us to verify that, under $\mathbb{P}_{W_{s}}^{*}, A_{\infty}^{\nu, \Omega}=\sum A_{\infty}^{\nu, \Omega}\left(W^{i}\right)$, in the notation of this proposition, and so we get $\mathbb{N}_{t, x}$ a.e., $d A_{s}^{\nu, \Omega}$ a.e.,

Hence,

$$
\mathbb{E}_{W_{s}}^{*}\left(\exp -A_{\infty}^{\nu, \Omega}\right)=\exp -2 \int_{t}^{0} d r \mathbb{N}_{r, W_{s}(r)}\left(1-\exp -A_{\infty}^{\nu, \Omega}\right) .
$$

$$
\begin{aligned}
& u(t, x)=\mathbb{N}_{t, x}\left(\int_{0}^{\infty} d A_{s}^{\nu, \Omega} \exp -2 \int_{t}^{0} d r u\left(r, W_{s}(r)\right)\right) \\
& =\int \nu(d y) p_{-t}^{\Omega}(x, y) E_{x y}^{t 0, \Omega}\left(\exp -2 \int_{t}^{0} d r u(r, w(r))\right)
\end{aligned}
$$

by (6). The proof of (8) is then completed by routine calculations. Using simple properties of the law of the Brownian bridge, we get

$$
\begin{gathered}
u(t, x)=h(t, x)-2 \int \nu(d y) p_{-t}^{\Omega}(x, y) E_{x y}^{t 0, \Omega}\left(\int_{t}^{0} d r u(r, w(r)) \exp -2 \int_{r}^{0} d s u(s, w(s))\right) \\
=h(t, x)-2 \int \nu(d y) E_{t, x}\left(\int_{t}^{\tau} d r u\left(r, \xi_{r}\right) p_{-r}^{\Omega}\left(\xi_{r}, y\right) E_{\xi_{r} y}^{r 0, \Omega}\left(\exp -2 \int_{r}^{0} d s u(s, w(s))\right)\right) \\
=h(t, x)-2 E_{t, x}\left(\int_{t}^{\tau} d r u\left(r, \xi_{r}\right)^{2}\right),
\end{gathered}
$$

where in the last equality we used the previous displayed formula for $u$.

Since $h$ is space-time harmonic (for $\frac{\partial}{\partial t}+\frac{1}{2} \Delta$ ) in $D$, it is easy to verify that $u$ solves (4). The uniqueness of the nonnegative solution of (6) follows by the same arguments as in the uniqueness part of the proof of Proposition 4.1 in [13]. Alternatively, this uniqueness can also be obtained as a very special case of Theorem 1.1 in [6].

To prove the last assertion, we first assume that $\nu$ has a finite energy. Consider the case $\varphi=1$, and note that $u(t, x) \leq h(t, x) \leq \int \nu(d y) p_{-t}(x, y)$. It follows that

$$
\begin{gathered}
\int_{\Omega} d x E_{t, x}\left(\int_{t}^{\tau} d r u\left(r, \xi_{r}\right)^{2}\right) \leq \int_{\mathbb{R}^{d}} d x E_{t, x}\left(\int_{t}^{0} d r\left(\int \nu(d y) p_{-r}\left(\xi_{r}, y\right)\right)^{2}\right) \\
=\iint \nu(d y) \nu\left(d y^{\prime}\right) \int_{t}^{0} d r \int_{\mathbb{R}^{d}} d z p_{-r}(z, y) p_{-r}\left(z, y^{\prime}\right)
\end{gathered}
$$




$$
=\iint \nu(d y) \nu\left(d y^{\prime}\right) \int_{0}^{-2 t} d r p_{2 r}\left(y, y^{\prime}\right)
$$

which tends to 0 as $t \uparrow 0$ by the dominated convergence theorem. Hence,

$$
\lim _{t \uparrow 0} \int_{\Omega} d x u(t, x)=\lim _{t \uparrow 0} \int_{\Omega} d x h(t, x)=\langle\nu, 1\rangle .
$$

Then, it is sufficient to consider the case $0 \leq \varphi \leq 1$. In this case,

$$
\limsup _{t \uparrow 0} \int_{\Omega} d x \varphi(x) u(t, x) \leq \limsup _{t \uparrow 0} \int_{\Omega} d x \varphi(x) h(t, x)=\langle\nu, \varphi\rangle,
$$

and replacing $\varphi$ by $1-\varphi$ yields the desired result. Finally a straightforward monotonicity argument makes it possible to drop the finite energy assumption.

Remark: Dynkin and Kuznetsov [6] solve integral equations of a more general form than (6) in terms of additive functionals of superprocesses. As a consequence of their results ([6], Theorem 1.3), the condition that $\nu$ does not charge polar sets is necessary for the existence of a (nonnegative) solution of (6). A proof of this fact using the Brownian snake can also be given along the lines of the proof of Proposition 4.3 in [13].

\subsection{Singular solutions}

It is also easy to get a probabilistic representation for solutions that tend to infinity on a part of the boundary. We limit ourselves to a special case that will be needed later. Let $D=$ $(-\infty, 0) \times \Omega$ as previously. Define under $\mathbb{N}_{t, x}$ the graph of the Brownian snake in $D$ by

$$
\mathrm{g}^{D}=\left\{W_{s}(r) ; s \geq 0, t \leq r \leq \tau\left(W_{s}\right) \wedge \zeta_{s}\right\} .
$$

Note that the support of $X^{D}$ is contained in $g^{D} \cap \partial D$. Let $U$ be a measurable subset of $\partial D$, and let $\nu$ be a finite measure on $\mathbb{R}^{d}$ not charging polar sets. Write $A^{\nu}=A^{\nu}, \mathbb{R}^{d}$ for simplicity. The function

$$
u(t, x)=\mathbb{N}_{t, x}\left(g^{D} \cap U \neq \emptyset\right)+\mathbb{N}_{t, x}\left(g^{D} \cap U=\emptyset ; 1-\exp -A_{\infty}^{\nu}\right), \quad(t, x) \in D,
$$

is a solution of (4) in $D$. The easiest way to verify this fact is to use the special Markov property for the Brownian snake [13]. Note that $u(t, x)=\mathbb{N}_{t, x}\left(1-e^{-Z}\right)$, where $Z=A_{\infty}^{\nu}+$ $\infty \cdot 1_{\left\{\mathcal{G}^{D} \cap U \neq \emptyset\right\}}$. Let $D^{\prime}=(-\infty, a) \times \Omega^{\prime}$ be a subdomain of $D$, with $a<0$ and $\bar{\Omega}^{\prime} \subset \Omega$. Then if $\left(\omega_{i}, i \in I\right)$ denote the excursions of the Brownian snake outside $D^{\prime}$ (see [13]), it is easy to verify that $Z=\sum Z\left(\omega_{i}\right), \mathbb{N}_{t, x}$ a.e. for $(t, x) \in D$ (use (7) to deal with the additive functional part). The special Markov property then gives

$$
u(t, x)=\mathbb{N}_{t, x}\left(1-\exp -\left\langle X^{D^{\prime}}, u\right\rangle\right)
$$

and, as we saw previously, this implies that $u$ solves (4) in $D^{\prime}$.

Let $(r, y)$ belong to the relative interior of $U$ in $\partial D$. Suppose that either $r=0$ or $y$ is regular for $\Omega^{c}$ (with respect to Brownian motion). Then, by writing $u(t, x) \geq \mathbb{N}_{t, x}\left(1-\exp -n\left\langle X^{D}, 1_{U}\right\rangle\right)$, it is easy to verify that $u$ has boundary value $+\infty$ at $(r, y)$.

\section{The Trace of a Solution} tion 5 .

The next lemma is the key to the proof of Theorem 1. Recall the notation $u_{\nu, \Omega}$ from Proposi- 
Lemma 6: Let $u$ be a (nonnegative) solution of (4) in the domain $(-\infty, 0) \times \Omega$. Let $B$ be an open ball whose closure $\bar{B}$ is contained in $\Omega$. Suppose that there exists a neighborhood $B^{\prime}$ of $\bar{B}$ in $\Omega$ such that

$$
\liminf _{t \uparrow 0} \int_{B^{\prime}} u(t, y) d y<\infty .
$$

Then there exists a finite measure $\nu$ on $B$ not charging polar sets such that

$$
u_{\nu, B}(t, x) \leq u(t, x), \quad \forall(t, x) \in(-\infty, 0) \times B
$$

and, for every compact subset $K$ of $B$,

$$
\lim _{t \uparrow 0} \sup _{y \in K}\left|u(t, y)-u_{\nu, B}(t, y)\right|=0 .
$$

Proof: We may assume that $B^{\prime}$ is a ball and $\bar{B}^{\prime} \subset \Omega$. Choose a sequence $t_{n} \uparrow 0$ such that

$$
\sup _{n} \int_{B^{\prime}} u\left(t_{n}, y\right) d y<\infty
$$

We set $D_{n}=\left(-\infty, t_{n}\right) \times B, D_{n}^{\prime}=\left(-\infty, t_{n}\right) \times B^{\prime}, D=(-\infty, 0) \times B$ and $D^{\prime}=(-\infty, 0) \times B^{\prime}$. We also denote by $\tau_{n}, \tau_{n}^{\prime}$ and $\tau$ the first exit times from $D_{n}, D_{n}^{\prime}$ and $D$, respectively. For $(t, x) \in D_{n}^{\prime}$, set

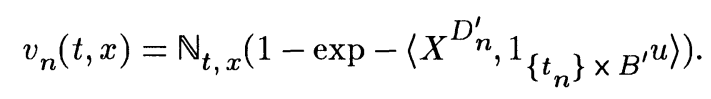

By the results recalled in Section 2.3, $v_{n}$ solves the integral equation

$$
v_{n}(t, x)+2 E_{t, x} \int_{t}^{\tau_{n}^{\prime}} v_{n}\left(r, \xi_{r}\right)^{2} d r=E_{t, x}\left(u\left(t_{n}, \xi_{t_{n}}\right) 1_{\left\{\tau_{n}^{\prime}=t_{n}\right\}}\right) .
$$

From (10), we get for every $(t, x) \in D^{\prime}$

and so

$$
\sup _{n: t_{n}>t} E_{t, x}\left(u\left(t_{n}, \xi_{t_{n}}\right) 1_{\left\{\tau_{n}^{\prime}=t_{n}\right\}}\right)<\infty
$$

$$
\sup _{n: t_{n}>t} E_{t, x} \int_{t}^{\tau_{n}^{\prime}} v_{n}\left(r, \xi_{r}\right)^{2} d r<\infty .
$$

Choose $r>0$ such that $B(y, r) \subset B^{\prime}$ if $y \in B$. Then, the "mean value property" of solutions of (4) gives for $(t, x) \in D_{n}$,

$$
\begin{aligned}
& u(t, x)=\mathbb{N}_{t, x}\left(1-\exp -\left\langle X^{\left.\left.D_{n}^{\prime}, u\right\rangle\right)}\right.\right.
\end{aligned}
$$

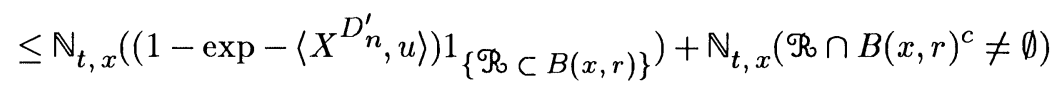

$$
\begin{aligned}
& \leq v_{n}(t, x)+c_{d} r^{-2}
\end{aligned}
$$

because on the event $\{\mathscr{B} \subseteq B(x, r)\}$, it is clear that $X^{D_{n}^{\prime}}$ is supported on $\left\{t_{n}\right\} \times B^{\prime}$. From the last bound, (11), and Fatou's lemma, we get for $(t, x) \in D$ that

$$
E_{t, x} \int_{t}^{\tau} u\left(r, \xi_{r}\right)^{2} d r<\infty
$$

We then set for $(t, x) \in D_{n}$

$$
u_{n}(t, x)=\mathbb{N}_{t, x}\left(1-\exp -\left\langle X^{D}, 1_{\left\{t_{n}\right\} \times B} u\right\rangle\right) .
$$


Note that $u_{n}(t, x) \leq \mathbb{N}_{t, x}\left(1-\exp -\left\langle X^{D_{n}}, u\right\rangle\right)=u(t, x)$. Furthermore, $u$ solves (4) in $D_{n}$ and satisfies the integral equation

$$
\begin{gathered}
u_{n}(t, x)+2 E_{t, x} \int_{t}^{\tau_{n}} d r u_{n}\left(r, \xi_{r}\right)^{2}=E_{t, x}\left(\left(1_{\left\{t_{n}\right\} \times B} u\right)\left(\tau_{n}, \xi_{\tau_{n}}\right)\right) \\
\quad=\int_{B} d y p_{t_{n}-t}^{B}(x, y) u\left(t_{n}, y\right) .
\end{gathered}
$$

From this integral equation, we easily see that

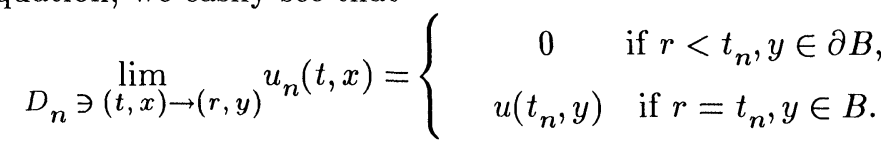

Since $u_{n+1} \leq u$ on $\left\{t_{n}\right\} \times B$, the maximum principle implies that $u_{n+1} \leq u_{n}$ in $D_{n}$. We can therefore set for $(t, x) \in D$,

$$
v(t, x)=\lim _{n \rightarrow \infty} \downarrow u_{n}(t, x)
$$

Replacing $\left(t_{n}\right)$ by a subsequence, we may assume that the measures $1_{B}(y) u\left(t_{n}, y\right) d y$ converge weakly to a finite measure $\eta(d y)$ on $\bar{B}$. We let $\nu$ be the restriction of $\eta$ on $B$. We can then pass to the limit $n \rightarrow \infty$ in the integral equation for $u_{n}$, using the weak convergence of the measures $1_{B}(y) u\left(t_{n}, y\right) d y$, the bound $u_{n} \leq u$, and (12) to justify dominated convergence. In the limit we get

$$
v(t, x)+2 E_{t, x} \int_{t}^{\tau} d r v\left(r, \xi_{r}\right)^{2}=\int_{B} \nu(d y) p_{-t}^{B}(x, y) .
$$

By Proposition 5, this exactly means that $v=u_{\nu, B}$. Furthermore, by the remark at the end of Subsection 2.4, the existence of a nonnegative solution to the previous integral equation implies that $\nu$ does not charge polar sets.

To verify (9), note that $\operatorname{supp} X^{D_{n}} \subset\left[t, t_{n}\right] \times \mathscr{R}_{[t, 0]}, \mathbb{N}_{t, x}$ a.e. Let $K$ be a compact subset of $B$, and let $r>0$ be such that $B(y, r) \subset B$ for every $y \in K$. By the previous observation, if $x \in K$ and $t<t_{n}$,

$$
\begin{aligned}
& u(t, x) \leq \mathbb{N}_{t, x}\left(\left(1-\exp -\left\langle X^{\left.\left.\left.D_{n}, u\right\rangle\right) 1_{\left\{\mathscr{R}_{[t, 0]}\right.} \subset B(x, r)\right\}}\right)+\mathbb{N}_{t, x}\left(\mathscr{R}_{[t, 0]} \cap B(x, r)^{c} \neq \emptyset\right)\right.\right.
\end{aligned}
$$

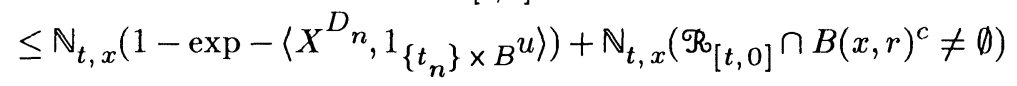

$$
\begin{aligned}
& =u_{n}(t, x)+\psi_{r}(-t) \text {. }
\end{aligned}
$$

By passing to the limit $n \rightarrow \infty$, we get $u(t, x) \leq u_{\nu, B}(t, x)+\psi_{r}(-t)$, for every $(t, x) \in(-\infty, 0) \times$ $K$. Recall from (5) that $\psi_{r}(-t) \downarrow 0$ as $t \uparrow 0$. Since we already know that $u_{\nu, B} \leq u_{n} \leq u$ in $D_{n}$, the proof of (9) is complete.

Proof of Theorem 1: Let $u$ be a solution of $(4)$ in $(-\infty, 0) \times \Omega$. Set

$$
\Lambda=\left\{y \in \Omega ; \forall r>0, \lim _{t \uparrow 0} \int_{B(y, r)} u(t, z) d z=\infty\right\} .
$$

Note that $\Lambda$ is a closed subset of $\Omega$. By Lemma 6 and the last assertion of Proposition 5 , for every $x \in \Omega \backslash \Lambda$, there exists an open ball $B_{x}$ centered at $x$, contained in $\Omega \backslash \Lambda$, and a finite measure $\nu_{B_{x}}$ on $B_{x}$ not charging polar sets such that

$$
\lim _{t \uparrow 0} \int_{B_{x}} d y u(t, y) \varphi(y)=\int \nu_{B_{x}}(d y) \varphi(y),
$$

for every $\varphi \in C_{c}\left(B_{x}\right)$. Standard arguments then give the existence of a Radon measure $\nu$ on $\Omega \backslash \Lambda$ 
not charging polar sets and such that

$$
\lim _{t \uparrow 0} \int_{\Omega \backslash \Lambda} d y u(t, y) \varphi(y)=\int \nu(d y) \varphi(y),
$$

for every $\varphi \in C_{c}(\Omega \backslash \Lambda)$. Theorem 1 follows.

Remark: The pair $(\Lambda, \nu)$ is called the trace of $\nu$. As a consequence of Lemma 6 , we also arrive at the following fact that will be needed alter. Let $u_{1}$ and $u_{2}$ be two solutions of (4) in $(-\infty, 0) \times \Omega_{1}$ and $(-\infty, 0) \times \Omega_{2}$, respectively, with respective traces $\left(\Lambda_{1}, \nu_{1}\right)$ and $\left(\Lambda_{2}, \nu_{2}\right)$. Suppose that there exists a ball $B$ such that $\bar{B} \subset\left(\Omega_{1} \backslash \Lambda_{1}\right) \cap\left(\Omega_{2} \backslash \Lambda_{2}\right)$ and the measures $\nu_{1}$ and $\nu_{2}$ coincide on a neighborhood of $\bar{B}$. Then,

$$
\lim _{t \uparrow 0} \sup _{x \in \bar{B}}\left|u_{1}(t, x)-u_{2}(t, x)\right|=0 .
$$

This follows from (9) by comparing $u_{1}$ and $u_{2}$ with the solution $u_{\nu, B^{\prime}}$, where $B^{\prime}$ is a suitable neighborhood of $\bar{B}$ and $\nu$ stands for the restriction of $\nu_{1}$, or $\nu_{2}$, on $B^{\prime}$.'

The proof of Proposition 2 depends on the following two lemmas.

Lemma 7: Let $(\Lambda, \nu)$ be the trace of a solution $u$ of $(4)$ in $(-\infty, 0) \times \Omega$. Property $(b)$ of Proposition 2 holds for the pair $(\Lambda, \nu)$.

Proof: Let $H$ be as in property (b), and suppose that $H \neq \emptyset$. Then, let $x \in H$ and let $B$ be an open ball centered at $x$ such that $\bar{B} \cap \Lambda$ is polar and $\nu\left(B^{\prime}\right)<\infty$ for a certain neighborhood $B^{\prime}$ of $\bar{B}$. The existence of $B$ follows from our assumptions on $H$. Denote by $\nu_{B^{\prime}}$ the restriction of $\nu$ on $B^{\prime}$ and write $A^{\nu} B^{\prime \prime} \mathbb{R}^{d}$. Finally, let $(\bar{B} \cap \Lambda)_{\varepsilon}$ denote the open $\varepsilon$-neighborhood of $\bar{B} \cap \Lambda$ in $\mathbb{R}^{d}$ and for $(t, y) \in D:=(-\infty, 0) \times B$, set

$$
\begin{gathered}
v_{\varepsilon}(t, y)=\mathbb{N}_{t, y}\left(\left\{\mathscr{R}_{[t, 0]} \cap B^{c} \neq \emptyset\right\} \cup\left\{\varphi_{0} \cap(\bar{B} \cap \Lambda)_{\varepsilon} \neq \emptyset\right\}\right) \\
+\mathbb{N}_{t, y}\left(\left\{\mathscr{R}_{[t, 0]} \cap B^{c}=\emptyset\right\} \cap\left\{\varphi_{0} \cap(\bar{B} \cap \Lambda)_{\varepsilon}=\emptyset\right\} ; 1-\exp -A_{\infty}^{\nu} B^{\prime}\right) .
\end{gathered}
$$

The function $v_{\varepsilon}$ is of the type considered in Subsection 2.5. Hence, $v_{\varepsilon}$ solves (4) in $D$. Furthermore, for $r<0, z \in \partial B$,

$$
\lim _{(t, y) \rightarrow(r, z)} v_{\varepsilon}(t, y)=\infty
$$

In addition, if $z \in(\bar{B} \cap \Lambda)_{\varepsilon} \cap \bar{B}$, a simple argument using (5) shows that

$$
\lim _{(t, y) \rightarrow(0, z)}\left(v_{\varepsilon}(t, y)-\mathbb{N}_{t, y}\left(\mathcal{G} \cap D^{c} \neq \emptyset\right)\right)=0,
$$

and because $\mathbb{N}_{t, y}\left(9 \cap D^{c} \neq \emptyset\right)$ is the maximal solution in $D$ (Dynkin [4]), we also have

$$
\limsup _{(t, y) \rightarrow(0, z)}\left(u(t, y)-v_{\varepsilon}(t, y)\right) \leq 0
$$

This inequality also holds if $z \in \bar{B} \backslash \Lambda$, from the trivial bound $\mathbb{N}_{t, y}\left(1-\exp -A_{\infty}^{\nu} B^{\prime}\right) \leq v_{\varepsilon}(t, y)$ and the remark following the proof of Theorem 1 .

By combining the previous observations, we have

$$
\limsup _{(t, y) \rightarrow(r, z)}\left(u(t, y)-v_{\varepsilon}(t, y)\right) \leq 0
$$

for every $(r, z) \in \partial D$. In particular, $u-v_{\varepsilon}$ is bounded above on the sets of the form $(a, 0) \times B$, and the maximum principle implies that $u \leq v_{\varepsilon}$ in $D$. By letting $\varepsilon \rightarrow 0$, we get

$$
u(t, y) \leq \mathbb{N}_{t, y}\left(\left\{\mathscr{R}_{[t, 0]} \cap B^{c} \neq \emptyset\right\} \cup\left\{\varphi_{0} \cap(\bar{B} \cap \Lambda) \neq \emptyset\right\}\right)
$$




$$
\begin{aligned}
& +\mathbb{N}_{t, y}\left(\left\{\mathscr{B}_{[t, 0]} \cap B^{c}=\emptyset\right\} \cap\left\{\varphi_{0} \cap(\bar{B} \cap \Lambda)=\emptyset\right\} ; 1-\exp -A_{\infty}^{\nu} B^{\prime}\right) \\
& \left.=\mathbb{N}_{t, y}\left(\mathscr{R}_{[t, 0]} \cap B^{c} \neq \emptyset\right\}+\mathbb{N}_{t, y}\left(\mathscr{R}_{[t, 0]} \cap B^{c}=\emptyset\right\} ; 1-\exp -A_{\infty}^{\nu} B^{\prime}\right),
\end{aligned}
$$

because the polar set $\bar{B} \cap \Lambda$ is not hit by $\varphi_{0}, \mathbb{N}_{t, y}$ a.e. This bound and (5) imply that

$$
\limsup _{(t, y) \rightarrow(0, z)}\left(u(t, y)-\mathbb{N}_{t, y}\left(1-\exp -A_{\infty}^{\nu} B^{\prime}\right)\right) \leq 0
$$

uniformly when $z$ varies over a compact subset of $B$. Clearly, this implies that $B \cap \Lambda=\emptyset$ which gives a contradiction.

Lemma 8: Let $\Lambda$ be a closed subset of $\mathbb{R}^{d}$ and let $v(t, y)=\mathbb{N}_{t, y}\left(\varphi_{0} \cap \Lambda \neq \emptyset\right)$. The set

is polar.

$$
H=\left\{y \in \Lambda, \exists r>0: \liminf _{t \uparrow 0} \int_{B(y, r)} v(t, z) d z<\infty\right\}
$$

Proof: It is relatively easy to give an analytic proof along the lines of the proof of Lemma 5.1 in [7]. We will give a probabilistic argument that does not use the connections with partial differential equations. It is sufficient to prove that, if $B$ is an open ball such that, for a certain sequence $t_{n} \uparrow 0$,

then $B \cap \Lambda$ is polar.

$$
\sup _{n} \int_{B} v\left(t_{n}, z\right) d z<\infty
$$

First note that, by the first-moment formula for $X_{t}$, if $t<t_{n}$ and $x \in \mathbb{R}^{d}$,

$$
\mathbb{N}_{t, x}\left(\left\langle X_{t_{n}}, 1_{B} v\left(t_{n}, \cdot\right)\right\rangle\right)=E_{t, x}\left(1_{B}\left(\xi_{t_{n}}\right) v\left(t_{n}, \xi_{t_{n}}\right)\right)
$$

and so by our assumption,

$$
\sup _{n: t_{n}<t} \mathbb{N}_{t, x}\left(\left\langle X_{t_{n}}, 1_{B} v\left(t_{n}, \cdot\right)\right\rangle\right)<\infty
$$

On the other hand, by the special Markov property [13], $\mathbb{N}_{t, x}\left(\left\langle X_{t_{n}}, 1_{B} v\left(t_{n}, \cdot\right)\right\rangle\right)$ is the expected number (under $\mathbb{N}_{t, x}$ ) of the "excursions" of the Brownian snake in $\left[t_{n}, \infty\right) \times \mathbb{R}^{d}$ that start from $\left\{t_{n}\right\} \times B$ and hit $\{0\} \times \Lambda$. Letting $n \rightarrow \infty$, we get from the previous bound that

$$
\mathbb{N}_{t, x}\left(\operatorname{Card}\left\{s \geq 0 ; \zeta_{s}=0, W_{s}(0) \in B \cap \Lambda\right\}\right)<\infty \text {. }
$$

This implies that the set $\left\{w ; \zeta_{w}=0, w(0) \in B \cap \Lambda\right\}$ is semipolar for the Brownian snake, hence also $M_{t, x}$-polar in the terminology of [9] (because the Brownian snake is a symmetric Markov process, see [9]). By the Dynkin result recalled at the end of Subsection 2.2, this is equivalent to saying that $B \cap \Lambda$ is polar.

Proof of Proposition 2: The necessity of condition (a) has already been established in the construction of the trace, and the necessity of (b) follows from Lemma 7. To prove the sufficiency of (a) and (b), we may clearly take $\Omega=\mathbb{R}^{d}$ (otherwise we construct a solution in $(-\infty, 0) \times \mathbb{R}^{d}$ with trace $\left(\Lambda \cup \Omega^{c}, \nu\right)$ and then we restrict it to $\left.(-\infty, 0) \times \Omega\right)$.

Let $\left(K_{n}\right)$ be an increasing sequence of compact subsets of $\Lambda^{c}$ such that $K_{n}$ is contained in the interior of $K_{n+1}$ and $\Lambda^{c}=\lim \uparrow K_{n}$ (thus any compact subset of $\Lambda^{c}$ is contained in $K_{n}$ for $n$ sufficiently large). Denote by $\nu_{n}$ the restriction of $\nu$ on $K_{n}$. Following Section 2, we can define for every $n$ the additive functional $A^{\nu_{n}}=A^{\nu_{n}, \mathbb{R}^{d}}$, and these additive functionals form an increasing sequence. We then set

$$
A_{\infty}^{\nu}=\lim \uparrow A_{\infty}^{\nu}
$$

It is easy to verify that this definition does not depend on the choice of the sequence $\left(K_{n}\right)$. Clearly $A_{\infty}^{\nu}$ may be infinite. However, on the event $\left\{\varphi_{0} \cap \Lambda=\emptyset\right\}$, we can find a (random) integer 
$n$ such that $\varphi_{0} \subset K$, and then $A_{\infty}^{\nu}=A_{\infty}^{\nu}<\infty$.

We then claim that

$$
u(t, x)=\mathbb{N}_{t, x}\left(\varphi_{0} \cap \Lambda \neq \emptyset\right)+\mathbb{N}_{t, x}\left(\varphi_{0} \cap \Lambda=\emptyset ; 1-\exp -A_{\infty}^{\nu}\right)
$$

is a solution with trace $(\Lambda, \nu)$. The fact that $u$ solves (4) follows from Subsection 2.5. Then, let $B$ be an open ball such that $\bar{B} \subset \Lambda^{c}$ and let $n$ be such that $B \subset K_{n}$. From formulas (13) and (5) it easily follows that

$$
\lim _{t \uparrow 0}\left(u(t, x)-\mathbb{N}_{t, x}\left(1-\exp -A_{\infty}^{\nu}\right)\right)=0
$$

uniformly when $x$ varies on a compact subset of $B$. It follows that if $(\tilde{\Lambda}, \tilde{\nu})$ is the trace of $u$, $\tilde{\Lambda} \subseteq \underset{\sim}{\Lambda}$, and $\nu$ is the restriction of $\tilde{\nu}$ on $\Lambda^{c}$. To complete the proof, we need only verify that $\Lambda=\tilde{\Lambda}$. However, the set $\Lambda \backslash \tilde{\Lambda}$ contains no explosion point of $\widetilde{\nu}$, hence no explosion point of $\nu$. Furthermore, $\Lambda \backslash \tilde{\Lambda}$ is polar by Lemma 8 . By the property (b) of the pair $(\Lambda, \nu)$, we conclude that $\Lambda \backslash \tilde{\Lambda}=\emptyset$.

Remark: The previous argument is very similar to the proof of Theorem 5.2 in [7].

Proof of Proposition 3: We first check that the function $u$ defined by (13) is the maximal solution with trace $(\Lambda, \nu)$. For $\varepsilon>0$, let $\Lambda_{\varepsilon}$ be the open $\varepsilon$-neighborhood of $\Lambda$ and let

$$
\begin{gathered}
u_{\varepsilon}(t, x)=\mathbb{N}_{t, x}\left(\left\{\mathscr{R}_{[0, t]} \cap B\left(0, \varepsilon^{-1}\right)^{c} \neq \emptyset\right\} \cup\left\{\varphi_{0} \cap \Lambda_{\varepsilon} \neq \emptyset\right\}\right) \\
+\mathbb{N}_{t, x}\left(\left\{\mathscr{R}_{[0, t]} \subset B\left(0, \varepsilon^{-1}\right)\right\} \cap\left\{\varphi_{0} \cap \Lambda_{\varepsilon}=\emptyset\right\} ; 1-\exp -A_{\infty}^{\nu}\right) .
\end{gathered}
$$

From Subsection 2.5, $u_{\varepsilon}$ solves (4) in $(-\infty, 0) \times B\left(0, \varepsilon^{-1}\right)$, and it is clear that $u_{\varepsilon} \downarrow u$ as $\varepsilon \downarrow 0$. Let $v$ be another solution with trace $(\Lambda, \nu)$. Arguing as in the proof of Lemma 7, it is easy to see from the maximum principle that $v \leq u_{\varepsilon}$ in $(-\infty, 0) \times B\left(0, \varepsilon^{-1}\right)$. Letting $\varepsilon \rightarrow 0$, we conclude that $v \leq u$.

Suppose that $\Lambda$ is polar. Then, we can write

$$
u(t, x)=\mathbb{N}_{t, x}\left(1-\exp -A_{\infty}^{\nu}\right),
$$

because $\mathbb{N}_{t, x}\left(\varphi_{0} \cap \Lambda \neq \emptyset\right)=0$. Let $v$ be another solution with trace $(\Lambda, \nu)$ and let the sequence $\left(K_{n}, \nu_{n}\right)$ be as in the previous proof. By the remarks following the proof of Theorem 1, it is clear that for every $y \in K_{n}$,

Hence,

$$
\lim _{(t, x) \rightarrow(0, y)}\left|v(t, x)-\mathbb{N}_{t, x}\left(1-\exp -A_{\infty}^{\nu} n+1\right)\right|=0 .
$$

$$
\limsup _{(t, x) \rightarrow(0, y)}\left(\mathbb{N}_{t, x}\left(1-\exp -A_{\infty}^{\nu}\right)-v(t, x)\right) \leq 0 .
$$

The same bound holds trivially true when $y \notin K_{n}$, because then, by (5),

$$
\lim _{(t, x) \rightarrow(0, y)} \mathbb{N}_{t, x}\left(1-\exp -A_{\infty}^{\nu}\right)=0
$$

Thus (14) holds for every $y \in \mathbb{R}^{d}$. Let $B_{R}=B(0, R)$. Bound (14) is valid a fortiori for $y \in \bar{B}_{R}$ if we replace $A^{\nu} n$ by $A_{\infty}^{\nu_{n}, B_{R}} \leq A_{\infty}^{\nu_{n}}$. The maximum principle then gives for every $t<0, y \in B_{R}$,

$$
v(t, y) \geq \mathbb{N}_{t, y}\left(1-\exp -A_{\infty}^{\nu_{n}, B_{R}}\right) .
$$

By letting $R \rightarrow \infty$, we get $v(t, y) \geq \mathbb{N}_{t, y}\left(1-\exp -A_{\infty}^{\nu}\right)$ and by letting $n \rightarrow \infty$, we arrive at $v \geq u$, which implies $v=u$.

Finally, suppose that $d \geq 2$ and that $(\Lambda, 0)$ is a possible trace, with $\Lambda \neq \emptyset$. By property (b) of Proposition 2, for every $y \in \Lambda$ and $r>0$, the set $\Lambda \cap B(y, r)$ is not polar. Choose a sequence $\left(y_{n}\right)_{n \geq 1}$ dense in $\Lambda$, and for every $n$ a decreasing sequence $\left(r_{n}^{p}\right)_{p \geq 1}$ of positive numbers, and set for every $p \geq 1$ 


$$
\Lambda_{p}=\bigcup_{n=1}^{\infty}\left(\Lambda \cap B\left(y_{n}, r_{n}^{p}\right)\right)
$$

If $u_{p}(t, x)=\mathbb{N}_{t, x}\left(\varphi_{0} \cap \Lambda_{p} \neq \emptyset\right)$, then $u_{p}$ is a solution of (4) in $(-\infty, 0) \times \mathbb{R}^{d}$ (cf. Subsection 2.5). By comparing $u_{p}$ with the function $\mathbb{N}_{t, x}\left(\varphi_{0} \cap \Lambda \neq \emptyset\right)$ near points $\left(0, y_{n}\right)$, and using the fact that the sequence $\left(y_{n}\right)$ is dense in $\Lambda$, one easily concludes that the trace of $u_{p}$ is $(\Lambda, 0)$. We claim that, for a suitable choice of the numbers $r_{n}^{p}$, infinitely many of the functions $u_{p}$ must be different. To see this fix a point $\left(t_{0}, x_{0}\right)$. From the estimates for hitting probabilities of balls found in [1] (Theorem 3.1), if $d \geq 3$ and in [11] if $d=2$, we can choose each number $r_{n}^{p}$ small enough to make

$$
\mathbb{N}_{t_{0}, x_{0}}\left(\varphi_{0} \cap B\left(y_{n}, r_{n}^{p}\right) \neq \emptyset\right)
$$

as small as desired. Hence, by a suitable choice of the numbers $r_{n}^{p}$, we can construct the sets $\Lambda_{p}$ so that $u_{p}\left(t_{0}, x_{0}\right)$ tends to 0 as $p \rightarrow \infty$. This completes the proof.

\section{The One-Dimensional Case}

In this section, we prove Theorem 4 . We will rely on Theorem 1 , although a direct argument can also be given along the lines of [14].

Let $t>0, x \in \mathbb{R}$. Under $\mathbb{N}_{t, x}$, for every $r>t$, the measure $X_{r}$ is absolutely continuous and, more precisely, $X_{r}(d y)=Y_{r}(y) d y$, where the process $\left(Y_{r}(y),(r, y) \in(t, \infty) \times \mathbb{R}\right)$ is continuous. This follows from Theorem 1 in Sugitani [18] and the connections between super-Brownian motion and the Brownian snake.

Note that there are no nonempty polar sets in dimension 1 , so that any pair $(\Lambda, \nu)$, where $\Lambda$ is a closed subset of $\mathbb{R}$ and $\nu$ a Radon measure on $\Lambda^{c}$, is a possible trace. To prove Theorem 4, it suffices to verify that, if $u$ is a solution of $(4)$ in $(-\infty, 0) \times \mathbb{R}$ with trace $(\Lambda, \nu)$ then $u$ can be represented in the form

$$
u(t, x)=\mathbb{N}_{t, x}\left(\varphi_{0} \cap \Lambda \neq \emptyset\right)+\mathbb{N}_{t, x}\left(\varphi_{0} \cap \Lambda=\emptyset ; 1-\exp -\int \nu(d y) Y_{0}(y)\right)
$$

As was recalled in Subsection 2.3, we can write for $t<r<0$,

$$
u(t, x)=\mathbb{N}_{t, x}\left(1-\exp -\left\langle X_{r}, u(r, \cdot)\right\rangle\right)=\mathbb{N}_{t, x}\left(1-\exp -\int d y Y_{r}(y) u(r, y)\right) .
$$

Passing to the limit $r \uparrow 0$ in this formula we see that (15) follows from the below lemma.

Lemma 9: (a) $\mathbb{N}_{t, x}$ a.e. on the set $\left\{\varphi_{0} \cap \Lambda=\emptyset\right\}$ it holds true that

$$
\lim _{r \uparrow 0} \int d y Y_{r}(y) u(r, y)=\int \nu(d y) Y_{0}(y) .
$$

(b) $\mathbb{N}_{t, x}$ a.e. on the set $\left\{\varphi_{0} \cap \Lambda \neq \emptyset\right\}$,

$$
\lim _{r \uparrow 0} \int d y Y_{r}(y) u(r, y)=\infty
$$

Proof: Part (a) is easy to prove (in fact, much easier to prove than the corresponding statement in the elliptic case [14]). We know that $\varphi_{0}$ is compact. Hence, on the event $\left\{\varphi_{0} \cap \Lambda=\emptyset\right\}$, the function $y \rightarrow Y_{0}(y)$ is continuous with compact support contained in $\mathbb{R} \backslash \Lambda$. Because 0 is a.e. not a time of discontinuity of the mapping $r \rightarrow \varphi_{r}$ (see Perkins [17] or the last section of [8]), on the same event we can find $r_{0}<0$ and a compact subset $K$ of $\mathbb{R} \backslash \Lambda$ such that, for every $r \in\left[r_{0}, 0\right]$, the function $y \rightarrow Y_{r}(y)$ is supported on $K$. Then, if $r_{0} \leq r<0$,

$$
\left.\mid \int d y Y_{r}(y) u(r, y)\right)-\int \nu(d y) Y_{0}(y) \mid
$$




$$
\leq\left(\sup _{y \in K}\left|Y_{r}(y)-Y_{0}(y)\right|\right) \int_{K} d y u(r, y)+\left|\int d y u(r, y) Y_{0}(y)-\int \nu(d y) Y_{0}(y)\right|,
$$

which converges to 0 by Theorem 1 and the joint continuity of $Y_{r}(y)$.

Le us now prove part (b) of the lemma. From the definition of the trace and the joint continuity of $Y_{r}(y)$, it suffices to verify that, a.e. on $\left\{\varphi_{0} \cap \Lambda \neq \emptyset\right\}$, there exists a point $z \in \Lambda$ such that $Y_{0}(z)>0$. We will prove that this holds for $z=W_{T}(0)$, where

$$
T=\inf \left\{s ; \zeta_{s}=0, W_{s}(0) \in \Lambda\right\}
$$

is a stopping time that is finite a.e. on $\left\{\varphi_{0} \cap \Lambda \neq \emptyset\right\}$.

We have to show that

$$
\mathbb{N}_{t, x}\left(T<\infty, Y_{0}\left(W_{T}(0)\right)=0\right)=0 .
$$

By the strong Markov property at $T$, we have

$$
\mathbb{N}_{t, x}\left(T<\infty, Y_{0}\left(W_{T}(0)\right)=0\right)=\mathbb{N}_{t, x}\left(T<\infty, \mathbb{P}_{W_{T}}^{*}\left(Y_{0}\left(W_{0}(0)\right)=0\right)\right)
$$

(To see that the definition of $Y_{0}$ still makes sense under $\mathbb{P}_{w}^{*}$, use Proposition 2.5 of [10].)

We will prove that, if $w:[t, 0] \rightarrow \mathbb{R}$ is a finite path over the time interval $[t, 0]$, then $\mathbb{P}_{w}^{*}\left(Y_{0}(w(0))=0\right)=0$ as soon as, for a certain constant $A>0$,

$$
\operatorname{Card}\left\{n ;\left\{w(r),-2^{-n} \leq r \leq 0\right\} \subset B\left(w(0), A 2^{-n / 2}\right)\right\}=\infty .
$$

To this end, note that, by Proposition 2.5 of [10],

$$
\begin{aligned}
& \mathbb{P}_{w}^{*}\left(Y_{0}(w(0))=0\right)=\exp -2 \int_{t}^{0} d r \mathbb{N}_{r, w(r)}\left(Y_{0}(w(0))>0\right) \\
& \leq \prod_{n=n_{0}}^{\infty} \exp -2 \int_{-2}^{-2^{-n-1}} d r \mathbb{N}_{r, w(r)}\left(Y_{0}(w(0))>0\right),
\end{aligned}
$$

where $n_{0}$ is the first integer such that $t \leq-2^{-n_{0}}$.

Let $y, z \in \mathbb{R}$ and $r<0$ such that $-2^{-n} \leq r \leq-2^{-n-1}$ and $|y-z| \leq A 2^{-n / 2}$. By the Cauchy-Schwarz inequality,

$$
\mathbb{N}_{r, y}\left(Y_{0}(z)>0\right) \geq \frac{\left(\mathbb{N}_{r, y}\left(Y_{0}(z)\right)\right)^{2}}{\mathbb{N}_{r, y}\left(Y_{0}(z)^{2}\right)}
$$

Formulas for the moments of $Y_{0}$ are easily derived from the well-known formulas for the moments of $X_{t}$ :

$$
\mathbb{N}_{r, y}\left(Y_{0}(z)\right)=p_{-r}(y, z), \quad \mathbb{N}_{r, y}\left(Y_{0}(z)^{2}\right)=4 \int_{r}^{0} d \rho \int_{\mathbb{R}} d v p_{\rho-r}(y, v) p_{-\rho}(v, z)^{2} .
$$

These formulas and our assumptions on $y, z, r$ easily lead to the existence of two positive constants $C_{1}$ and $C_{2}$ depending only on $A$, such that

$$
\mathbb{N}_{r, y}\left(Y_{0}(z)\right) \geq C_{1} 2^{-n / 2}, \quad \mathbb{N}_{r, y}\left(Y_{0}(z)^{2}\right) \leq C_{2}
$$

It follows that $\mathbb{N}_{r, y}\left(Y_{0}(z)>0\right) \geq C_{3} 2^{-n}$, with $C_{3}=C_{2}^{-1} C_{1}^{2}$. By applying this bound to the previous formula for $\mathbb{P}_{w}^{*}\left(Y_{0}(w(0))=0\right)$, we get

$$
\mathbb{P}_{w}^{*}\left(Y_{0}(w(0))=0\right) \leq \exp \left(-C_{3} \operatorname{Card}\left\{n \geq n_{0} ;\left\{w(r),-2^{-n} \leq r \leq 0\right\} \subset B\left(w(0), A 2^{-n / 2}\right)\right\}\right)
$$

and, consequently, $\mathbb{P}_{w}^{*}\left(Y_{0}(w(0))=0\right)=0$ by $(17)$.

To get (16) and complete the proof, we need only validate (17), $\mathbb{N}_{t, x}$ a.e. on $\{T<\infty\}$ for the 
path $w=W_{T}$. However, this follows from the results of [11], which show that one can fix a deterministic constant $A$ such that (17) holds simultaneously for all paths $W_{s}$ such that $\zeta_{s}=0$. This completes the proof of Lemma 9 and of Theorem 4.

\section{Acknowledgements}

The author thanks E.B. Dynkin and S.E. Kuznetsov for letting him know about their recent unpublished work.

\section{References}

[1] Dawson, D.A., Iscoe, I. and Perkins, E.A., Super-Brownian motion: Path properties and hitting probabilities, Probab. Th. Rel. Fields 83 (1989), 135-205.

[2] Dhersin, J.S. and Le Gall, J.F., Wiener's test for super-Brownian motion and for the Brownian snake, Probab. Th. Rel. Fields (1997), to appear.

[3] Dynkin, E.B., A probabilistic approach to one class of nonlinear differential equations, Probab. Th. Rel. Fields 89 (1991), 89-115.

[4] Dynkin, E.B., Superdiffusions and parabolic nonlinear differential equations, Ann. Probab. 20 (1992), 942-962.

[5] Dynkin, E.B., Superprocesses and partial differential equations, Ann. Probab. 21 (1993), $1185-1262$.

[6] Dynkin, E.B. and Kuznetsov, S.E., Nonlinear parabolic p.d.e. and additive functionals of superdiffusions, Ann. Probab. (1997), to appear.

[7] Dynkin, E.B. and Kuznetsov, S.E., Trace on the boundary for solutions of nonlinear differential equations (1996), preprint.

[8] Le Gall, J.F., Brownian excursions, trees and measure-valued branching processes, Ann. Probab. 19 (1991), 1399-1439.

[9] Le Gall, J.F., A class of path-valued Markov processes and its applications to superprocesses, Probab. Th. Rel. Fields 95 (1993), 25-46.

[10] Le Gall, J.F., A path-valued Markov process and its connections with partial differential equations, In: Proc. First European Congress of Math. Vol. II (1994), Birkhäuser, Boston, 185-212.

[11] Le Gall, J.F., A lemma on super-Brownian motion with some applications, Festschrift in honor of E.B. Dynkin (ed. by M.I. Freidlin) (1994), Birkhäuser, Boston, 237-251.

[12] Le Gall, J.F., Hitting probabilities and potential theory for the Brownian path-valued process, Ann. Inst. Fourier 44 (1994), 277-306.

[13] Le Gall, J.F., The Brownian snake and solutions of $\Delta u=u^{2}$ in a domain, Probab. Th. Rel. Fields 102 (1995), 393-432.

[14] Le Gall, J.F., A probabilistic Poisson representation for positive solutions of $\Delta u=u^{2}$ in a domain, Comm. Pure Appl. Math. (1997), in press.

[15] Marcus, M. and Véron, L., Trace au bord des solutions positives d'équations elliptiques non linéaires, C.R. Acad. Sci. Paris, Série I 321 (1995), 179-184.

[16] Marcus, M. and Véron, L., Trace au bord des solutions positives d'équations elliptiques et paraboliques non linéaires. Résultats d'existence et d'unicité, C.R. Acad. Sci. Paris, Série I 323 (1996), 603-608.

[17] Perkins, E.A., Polar sets and multiple points for super-Brownian motion, Ann. Probab. 18 (1990), 453-491.

[18] Sugitani, S., Some properties for the measure-valued branching diffusion, J. Math. Soc. Japan 41 (1989), 437-462. 


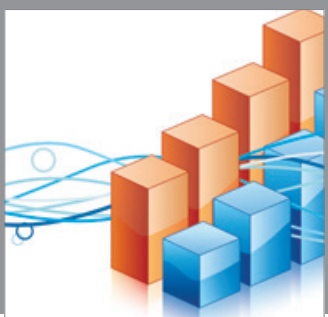

Advances in

Operations Research

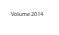

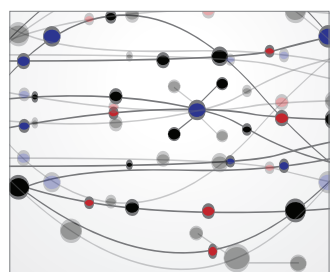

\section{The Scientific} World Journal
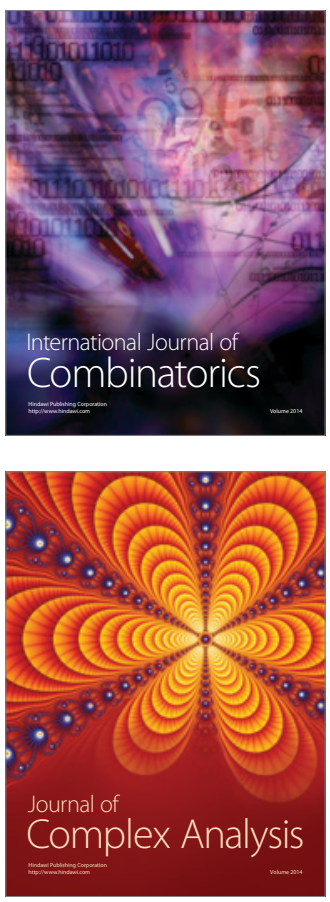

International Journal of

Mathematics and

Mathematical

Sciences
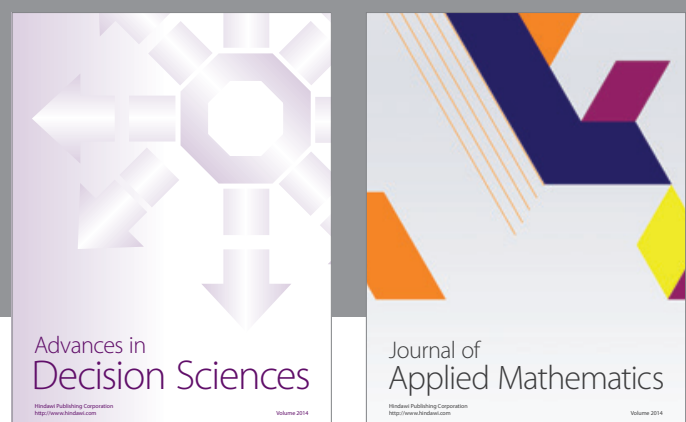

Journal of

Applied Mathematics
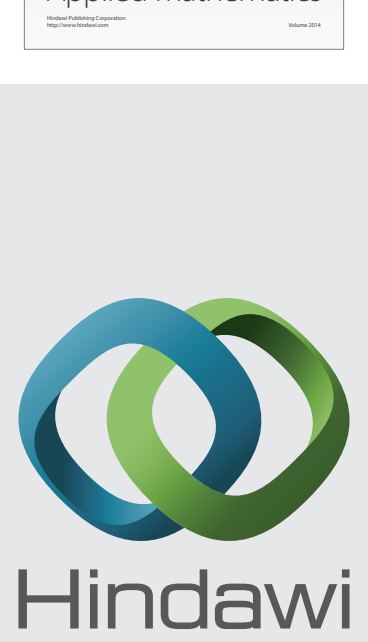

Submit your manuscripts at http://www.hindawi.com
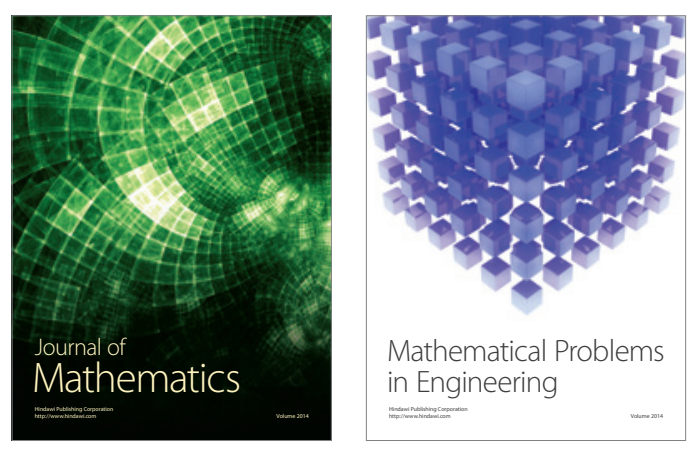

Mathematical Problems in Engineering
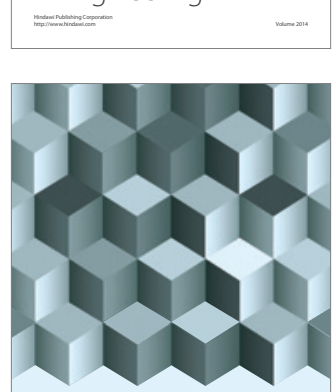

Journal of

Function Spaces
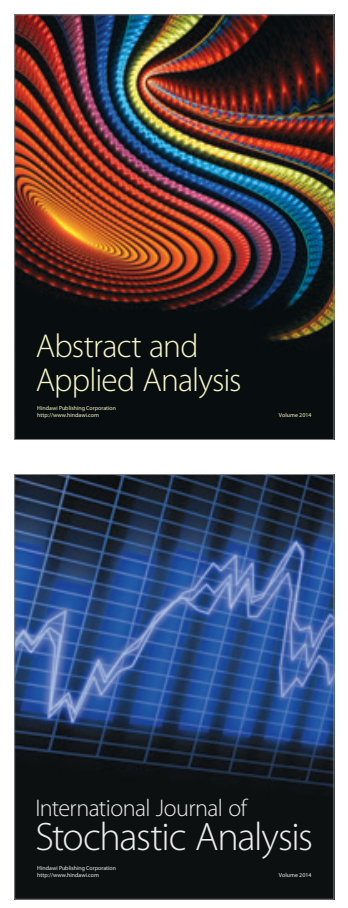

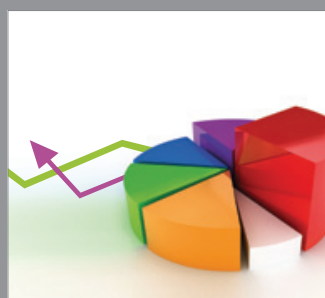

ournal of

Probability and Statistics

Promensencen
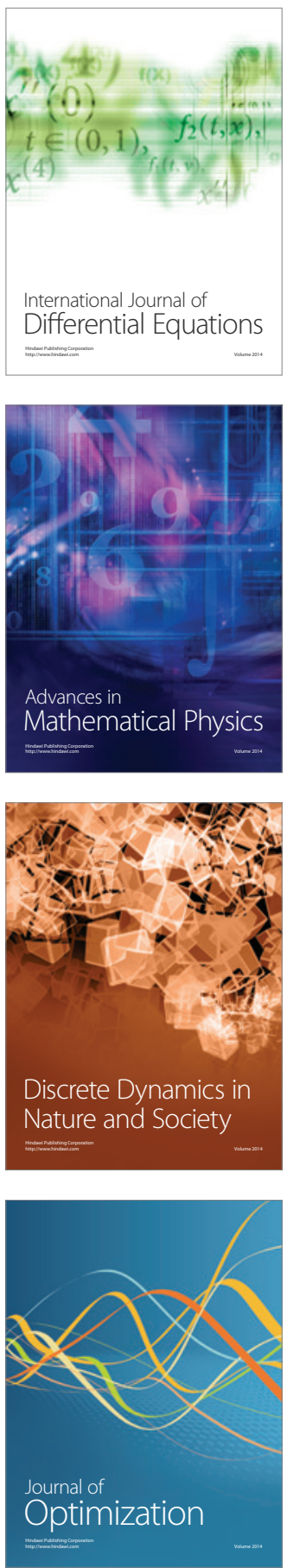